\title{
Streaming Potential in Unconsolidated Samples Saturated with Monovalent Electrolytes
}

\author{
Luong Duy Thanh ${ }^{1, *}$, Rudolf Sprik ${ }^{2}$ \\ ${ }^{I}$ Thuy Loi University, 175 Tay Son, Dong Da, Hanoi, Vietnam \\ ${ }^{2}$ Van der Waals-Zeeman Institute, University of Amsterdam, The Netherlands \\ Received 30 July 2017 \\ Revised 22 September 2017; Accepted 16 October 2017
}

\begin{abstract}
The streaming potential coefficient of liquid-rock systems is theoretically a very complicated function depending on many parameters including temperature, fluid concentration, fluid $\mathrm{pH}$, as well as rock parameters such as porosity, grain size, pore size, and formation factor etc. At a given porous media, the most influencing parameter is the fluid conductivity or electrolyte concentration. Therefore, it is useful to have an empirical relation between the streaming potential coefficient and electrolyte concentration. In this work, the measurements of the streaming potential for four unconsolidated samples (sandpacks) saturated with four monovalent electrolytes at six different electrolyte concentrations have been performed. From the measured streaming potential coefficient, the empirical expression between the streaming potential coefficient and electrolyte concentration is obtained. The obtained expression is in good agreement with those available in literature. Additionally, it is seen that the streaming potential coefficient depends on types of cation in electrolytes and on samples. The dependence of the streaming potential coefficient on types of cation is qualitatively explained by the difference in the binding constant for cation adsorption on the silica surfaces. The dependence of the streaming potential coefficient on samples is due to the variation of effective conductivity and the zeta potential between samples.
\end{abstract}

Keywords: Streaming potential coefficient, zeta potential, porous media, sands.

\section{Introduction}

The streaming potential is induced by the relative motion between the fluid and the solid surface. In porous media such as rocks, sands or soils, the electric current density, linked to the ions within the fluid, is coupled to the fluid flow. Streaming potential plays an important role in geophysical applications. For example, the streaming potential is used to map subsurface flow and detect

\footnotetext{
* Corresponding author. Tel.: 84-936946975.

Email: luongduythanh2003@yahoo.com

https//doi.org/ 10.25073/2588-1124/vnumap.4213
} 
subsurface flow patterns in oil reservoirs [e.g., 1]. Streaming potential is also used to monitor subsurface flow in geothermal areas and volcanoes [e.g., 2, 3]. Monitoring of streaming potential anomalies has been proposed as a means of predicting earthquakes [e.g., 4, 5] and detecting of seepage through water retention structures such as dams, dikes, reservoir floors, and canals [6].

The streaming potential coefficient (SPC) is a very important parameter, since this parameter controls the amount of coupling between the fluid flow and the electric current flow in porous media. The SPC of liquid-rock systems is theoretically a very complicated function depending on 18 fundamental parameters including temperature, pore fluid concentration, fluid $\mathrm{pH}$, as well as rock parameters such as porosity, grain size, pore size, and formation factor etc [e.g., 7, 8]. At a given porous media, the most influencing parameter is the fluid conductivity. Therefore, it is useful to have an empirical relation between the SPC and fluid conductivity or electrolyte concentration. For example, Jouniaux and Ishido [8] obtain an empirical relation to predict the SPC from fluid conductivity based on numerous measurements of the streaming potential on sand saturated by $\mathrm{NaCl}$ which have been published. By fitting experimental data collected for sandstone, sand, silica nanochannels, Stainton, and Fontainebleau with electrolytes of $\mathrm{NaCl}$ and $\mathrm{KCl}$, Jaafar et al. [9] obtain another empirical expression between the SPC and electrolyte concentration. However, experimental data sets they used for fitting are from different sources with dissimilar fluid conductivity, fluid $\mathrm{pH}$, temperature, mineral composition of porous media. All those dissimilarities may cause the empirical expressions less accurate. To critically seek empirical expressions to estimate the SPC from electrolyte concentration, we have carried out streaming potential measurements for a set of four sandpacks saturated by four monovalent electrolytes $(\mathrm{NaCl}, \mathrm{NaI}, \mathrm{KCl}$ and $\mathrm{KI})$ at six different electrolyte concentrations $\left(10^{-4} \mathrm{M}, 5.0 \times 10^{-4} \mathrm{M}, 10^{-3} \mathrm{M}, 2.5 \times 10^{-3} \mathrm{M}, 5.0 \times 10^{-3} \mathrm{M}\right.$, and $\left.10^{-2} \mathrm{M}\right)$.

From the measured SPC, we obtain the empirical expression between the SPC and electrolyte concentration. The obtained expression is in good agreement with those reported in $[8,9]$ in which the SPC in magnitude is inversely proportional to electrolyte concentration. Additionally, it is seen that the streaming potential coefficient depends on types of cation in electrolytes and on samples. The dependence of the streaming potential coefficient on types of cation is qualitatively explained by the difference in the binding constant for cation adsorption on the silica surfaces. The dependence of the streaming potential coefficient on samples is due to the variation of effective conductivity and the zeta potential between samples.

This paper includes five sections. Section 2 describes the theoretical background of streaming potential. Section 3 presents the experimental measurement. Section 4 contains the experimental results and discussion. Conclusions are provided in the final section.

\section{Theoretical background of streaming potential}

\subsection{Electrical double layer}

A porous medium is formed by mineral solid grains such as silicates, oxides, carbonates etc. When a solid grain surface is in contact with a liquid, it acquires a surface electric charge [12]. The surface charge repels ions in the electrolyte whose charges have the same sign as the surface charge (called the "coions") and attracts ions whose charges have the opposite sign (called the "counterions" and normally cations) in the vicinity of the electrolyte silica interface. This leads to the charge distribution known as the electric double layer (EDL) as shown in Fig. 1. The EDL is made up of the Stern layer, where cations are adsorbed on the surface and are immobile due to the strong electrostatic attraction, and the diffuse layer, where the ions are mobile. The distribution of ions and the electric potential 
within the diffuse layer is governed by the Poisson Boltzman (PB) equation which accounts for the balance between electrostatic and thermal diffusion forces [12]. The solution to the linear PB equation in one dimension perpendicular to a broad planar interface is well-known and produces an electric potential profile that decays approximately exponentially with distance as shown in Fig. 1. In the bulk liquid, the number of cations and anions is equal so that it is electrically neutral. The closest plane to the solid surface in the diffuse layer at which flow occurs is termed the shear plane or the slipping plane, and the electrical potential at this plane is called the zeta potential $(\zeta)$. The zeta potential plays an important role in determining the degree of coupling between the electric flow and the fluid flow in porous media. Most reservoir rocks have a negative surface charge and a negative zeta potential when in contact with ground water $[13,14]$. The characteristic length over which the EDL exponentially decays is known as the Debye length and is on the order of a few nanometers in most rock-water systems. The Debye length is a measure of the diffuse layer thickness; its value depends solely on the properties of the fluid and not on the properties of the solid surface [15] and is given by (for a symmetric, monovalent electrolyte such as $\mathrm{NaCl}$ )

$$
\chi_{d}=\sqrt{\frac{\varepsilon_{o} \varepsilon_{r} k_{b} T}{2000 N e^{2} C_{f}}},
$$

where $k_{b}$ is the Boltzmann's constant, $\varepsilon_{0}$ is the dielectric permittivity in vacuum, $\varepsilon_{r}$ is the relative permittivity of the fluid, $T$ is temperature (in $\mathrm{K}$ ), $e$ is the elementary charge, $N$ is the Avogadro's number and $C_{f}$ is the electrolyte concentration $(\mathrm{mol} / \mathrm{L})$.

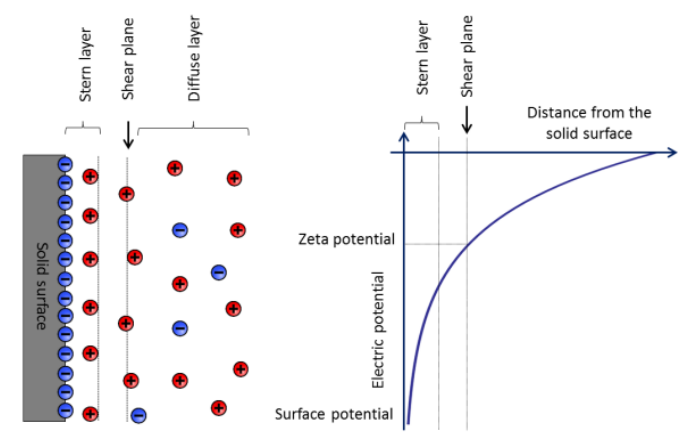

Figure 1. Stern model $[10,11]$ for the charge and electric potential distribution in the electric double layer at a solid-liquid interface. In this figure, the solid surface is negatively charged and the mobile counter-ions in the diffuse layer are positively charged (in most rock-water systems).

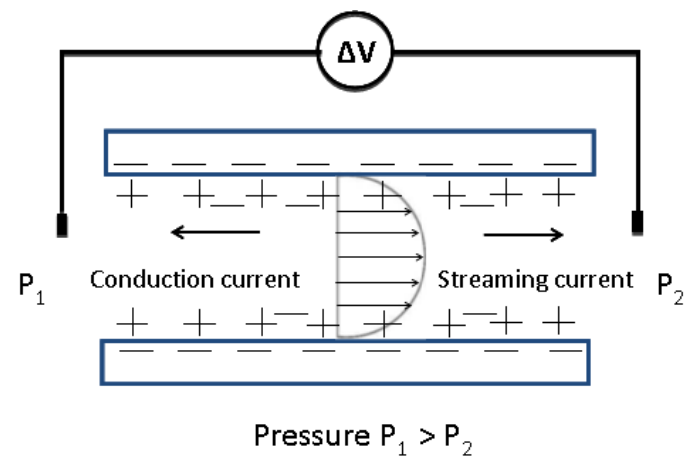

Figure 2. Development of streaming potential when an electrolyte is pumped through a capillary (a porous medium is made of an array of parallel capillaries). 


\subsection{Streaming potential}

The different flows (fluid flow, electrical current flow, heat flow etc.) are coupled by the general equation

$$
\mathbf{J}_{i}=\sum_{j=1}^{n} L_{i j} \mathbf{X}_{j}
$$
[16].

which links the forces $\mathbf{X}_{j}$ to the macroscopic fluxes $\mathbf{J}_{i}$ through transport coupling coefficients $L_{i j}$

Considering the coupling between the hydraulic flow and the electric current flow, assuming a constant temperature and no concentration gradients, the electric current density $\mathbf{J}_{\mathrm{e}}\left(\mathrm{A} / \mathrm{m}^{2}\right)$ and the flow of fluid $\mathbf{J}_{\mathrm{f}}(\mathrm{m} / \mathrm{s})$ can be written as the following coupled equation [e.g., 8]:

$$
\begin{aligned}
& \mathbf{J}_{\mathrm{e}}=-\sigma_{0} \nabla V-L_{e k} \nabla P . \\
& \mathbf{J}_{\mathrm{f}}=-L_{e k} \nabla V-\frac{k_{0}}{\eta} \nabla P,
\end{aligned}
$$

where $P$ is the pressure that drives the fluid flow $(\mathrm{Pa}), V$ is the electrical potential $(\mathrm{V}), \sigma_{0}$ is the bulk electrical conductivity, $k_{0}$ is the bulk permeability $\left(\mathrm{m}^{2}\right), \eta$ is the dynamic viscosity of the fluid (Pa.s), and $L_{e k}$ is the electrokinetic coupling (A.Pa ${ }^{-1} \cdot \mathrm{m}^{-1}$ ). The first term in Eq. (3) is the Ohm's law, and the second term in Eq. (4) is the Darcy's law. The coupling coefficient $L_{\mathrm{ek}}$ is the same in Eq. (3) and Eq. (4) because the coupling coefficients must satisfy the Onsager's reciprocal relation in the steady state. From these equations, it is possible to notice that even if no potential difference is applied $(\nabla V=0)$, then simply the presence of a pressure difference can produce an electric current. On the other hand, if no pressure difference is applied $(\nabla P=0)$, the presence of an electric potential difference can still generate a fluid flow by electroosmosis.

The SPC (V/Pa) is defined when the electric current density $\mathbf{J}_{\mathrm{e}}$ is zero, leading to

$$
C_{S}=\frac{\Delta V}{\Delta P}=-\frac{L_{e k}}{\sigma_{0}} \text {. }
$$

This coefficient can be measured by applying a pressure difference $\Delta P$ to a porous medium and by detecting the induced electric potential difference $\Delta V$ (see Fig. 2). The driving pressure induces a streaming current (second term in Eq. (3)) which is balanced by the conduction current (first term in Eq. (3)) which leads to the electric potential difference that can be measured. In the case of a unidirectional flow through a cylindrical saturated porous rock, this coefficient can be expressed as [e.g., 1, 4, 8]

$$
C_{S}=\frac{\varepsilon_{r} \varepsilon_{o} \zeta}{\eta \sigma_{e f f}}
$$

where $\sigma_{\text {eff }}$ is the effective conductivity, and $\zeta$ is the zeta potential. The effective conductivity includes the fluid conductivity and the surface conductivity. To characterize the relative contribution of the surface conductivity, the dimensionless quantity called the Dukhin number has been introduced [17]. The SPC can also be written as [e.g., 8, 18]

$$
C_{S}=\frac{\varepsilon_{r} \varepsilon_{o} \zeta}{\eta F \sigma_{r}}
$$


where $\sigma_{r}$ is the electrical conductivity of the sample saturated by a fluid with a conductivity of $\sigma_{f}$ and $F$ is the formation factor. The electrical conductivity of the sample can possibly include surface conductivity. If the fluid conductivity is much higher than the surface conductivity, the effective conductivity is approximately equal to the fluid conductivity, $\sigma_{e f f}=F \sigma_{r}=\sigma_{f}$ and the SPC becomes the well-known Helmholtz-Smoluchowski equation:

$$
C_{S}=\frac{\varepsilon_{r} \varepsilon_{o} \zeta}{\eta \sigma_{f}} .
$$

\section{Experiment}

Streaming potential measurements have been performed on four unconsolidated samples of sand particles with different diameters (See Table 1). The samples are made up of blasting sand particles obtained from Unicorn ICS BV Company. Four monovalent electrolytes $(\mathrm{NaCl}, \mathrm{NaI}, \mathrm{KCl}$ and $\mathrm{KI})$ are used with 6 different concentrations $\left(10^{-4} \mathrm{M}, 5.0 \times 10^{-4} \mathrm{M}, 10^{-3} \mathrm{M}, 2.5 \times 10^{-3} \mathrm{M}, 5.0 \times 10^{-3} \mathrm{M}\right.$, and $10^{-2}$ $\mathrm{M})$. All measurements are carried out at room temperature $\left(22 \pm 1^{\circ} \mathrm{C}\right)$.

\subsection{Sample assembly}

Samples are constructed by filling polycarbonate plastic tubes $(1 \mathrm{~cm}$ in inner diameter and $7.5 \mathrm{~cm}$ in length) successively with $2 \mathrm{~cm}$ thick layers of particles that are gently tamped down, and they are then shaken by a shaker (TIRA-model TV52110). Filter paper is used in both ends of the tube to retain the particles and is permeable enough to let the fluid pass through. The samples are flushed with distilled water to remove any powder or dust.

\subsection{Porosity, permeability, and formation factor measurements}

The porosity is measured by a simple method mentioned in [19] and reference therein. The sample is first dried in oven for 24 hours, then cooled to room temperature, and finally fully saturated with deionized water under vacuum. The sample is weighed before $\left(m_{d r y}\right)$ and after full saturation $\left(m_{w e t}\right)$ by a vacuum desiccator and the porosity is determined as

$$
\varphi=\frac{\left(m_{w e t}-m_{d r y}\right) / \rho}{A L},
$$

where $\rho$ is density of the water, $A$ and $L$ are the cross sectional area and the physical length of the samples, respectively. The measured porosity of the samples is 0.39 independently of the size of sand particles with an error of 5\%. This value is in good agreement with [20] for a random packing of spherical particles.

Table 1. Sample ID, diameter range, permeability $k_{o}$, porosity, formation factor $F$ and tortuosity $\alpha_{\infty}$.

\begin{tabular}{llllll}
\hline & Sample ID & Size $(\mu \mathrm{m})$ & $k_{o}\left(\mathrm{~m}^{2}\right)$ & $F$ & $\alpha_{\infty}$ \\
\hline 1 & S1 & $300-400$ & $13.2 \times 10^{-12}$ & 4.2 & 1.64 \\
2 & S2 & $200-300$ & $7.5 \times 10^{-12}$ & 4.0 & 1.56 \\
3 & S3 & $90-150$ & $3.8 \times 10^{-12}$ & 4.2 & 1.64 \\
4 & S4 & $0-50$ & $0.7 \times 10^{-12}$ & 4.3 & 1.68 \\
\hline
\end{tabular}




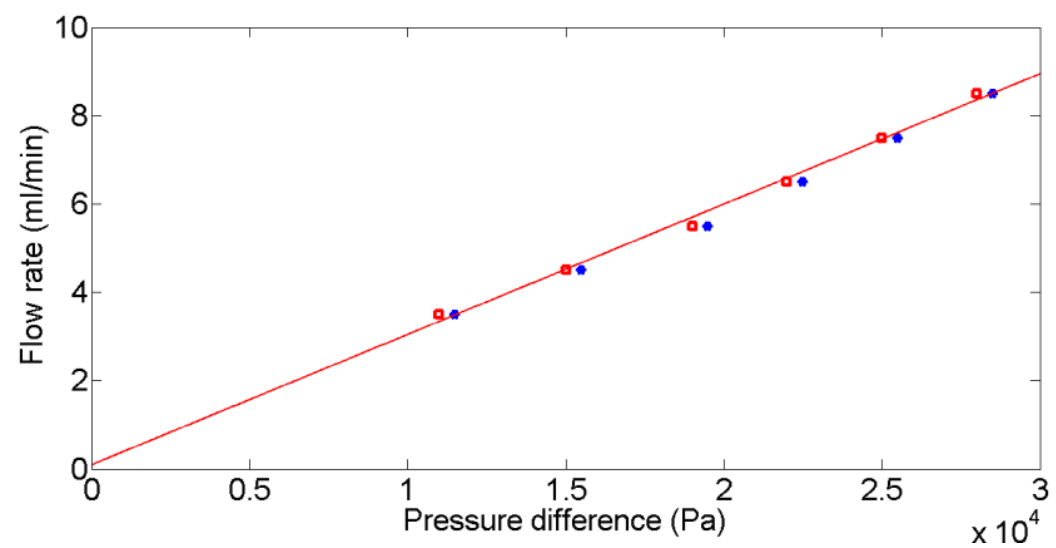

Figure 3. The flow rate against pressure difference. Two runs are shown for the sample S3.

Permeability is determined by a constant flow-rate experiment (see [19] and reference therein for more details). A high pressure pump (LabHut, Series III- Pump) ensures a constant flow through the sample, a high precision differential pressure transducer (Endress and Hauser Deltabar S PMD75) is used to measure the pressure drop. For low velocities Darcy's law holds

$$
Q_{f}=-\frac{k_{o} A}{\eta} \frac{\Delta P}{L}
$$

where $Q_{f}$ is the fluid volume flow rate, $k_{o}$ is the permeability, $\Delta P$ is the differential pressure imposed across the sample, $\eta$ is the viscosity of the fluid. The permeability is then determined from the slope of the flow rate - pressure graph (see Figure 3 for the sample S3, for example) with the knowledge of $L, A$ and $\eta\left(10^{-3} \mathrm{~Pa} . \mathrm{s}\right)$. The graph shows that there is a linear relationship between flow rate and pressure difference and Darcy's law is obeyed. Similarly, the permeability of other samples is obtained and reported in Table 1 with an error of $10 \%$.

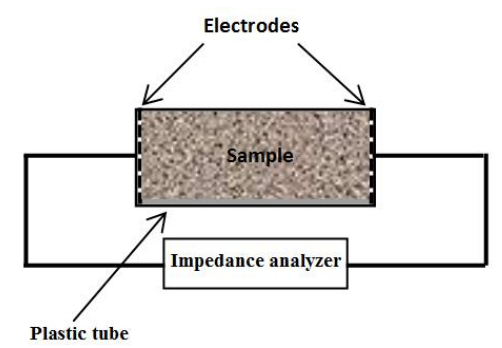

Figure 4. Schematic of the experimental setup to measure the saturated sample resistance.

Method of determining the formation factor and tortuosity is introduced in [19] and reference therein. The formation factor $F$ is defined as:

$$
F=\frac{\alpha_{\infty}}{\varphi}=\frac{\sigma_{f}}{\sigma_{r}}
$$


where $\alpha_{\infty}$ is the tortuosity, $\phi$ is the porosity of the sample, $\sigma_{f}$ is the electrical conductivity of the fluid directly measured by a conductivity meter (Consort C861) and $\sigma_{r}$ is the electrical conductivity of the fully saturated sample. Eq. (11) is only valid when surface conductivity effects become negligible ( $\sigma_{f}$ is higher than $0.60 \mathrm{~S} / \mathrm{m}$ as stated in [21] for silica-based samples). Schematic of the experimental setup to measure resistances of saturated samples is shown in Figure 4. The electrodes for the resistance measurements are silver meshes. The electrodes are placed on both sides against the sample that is fully saturated successively with a set of $\mathrm{NaCl}$ solutions with high conductivities. The sample resistance is measured by an impedance analyzer (Hioki IM3570) to calculate $\sigma_{r}$ with the knowledge of the geometry of the sample (the length and the diameter). From the measured values of $\sigma_{f}$ and $\sigma_{r}$, the formation factor and corresponding tortuosity are determined with an error of $6 \%$ and $9 \%$, respectively (see Table 1). The measured formation factors of the samples are the range from 4.0 to 4.3. According to Archie's law, $F=\varphi^{-m}$ in which $\varphi$ is the porosity of the sample and $m$ is the so called cementation exponent. For unconsolidated samples made of spherical particles, the exponent is around 1.5 [22]. Therefore, the measured formation factors are in good agreement with Archie's law.

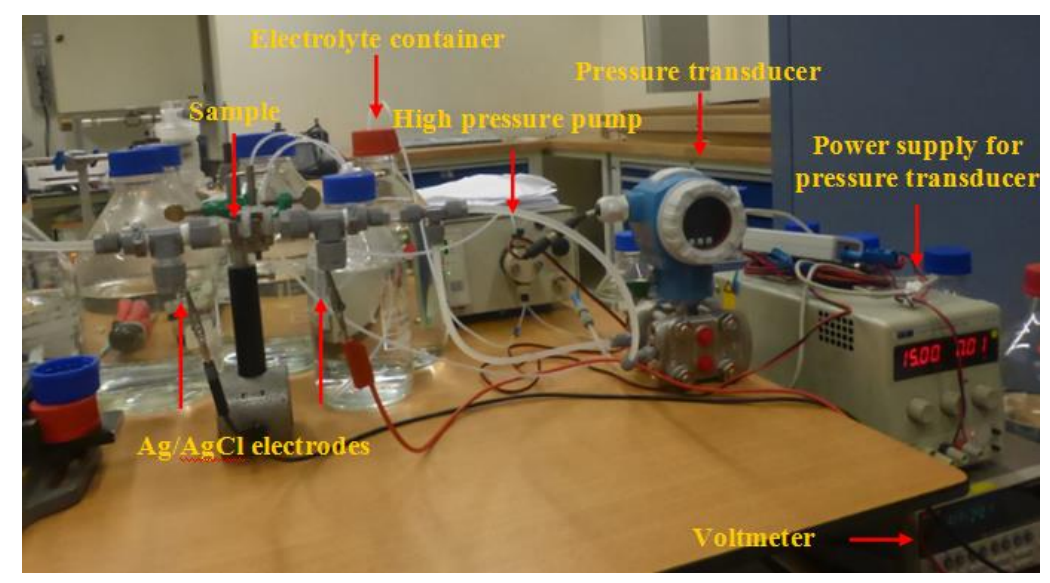

Figure 5. Experimental setup for streaming potential measurements.

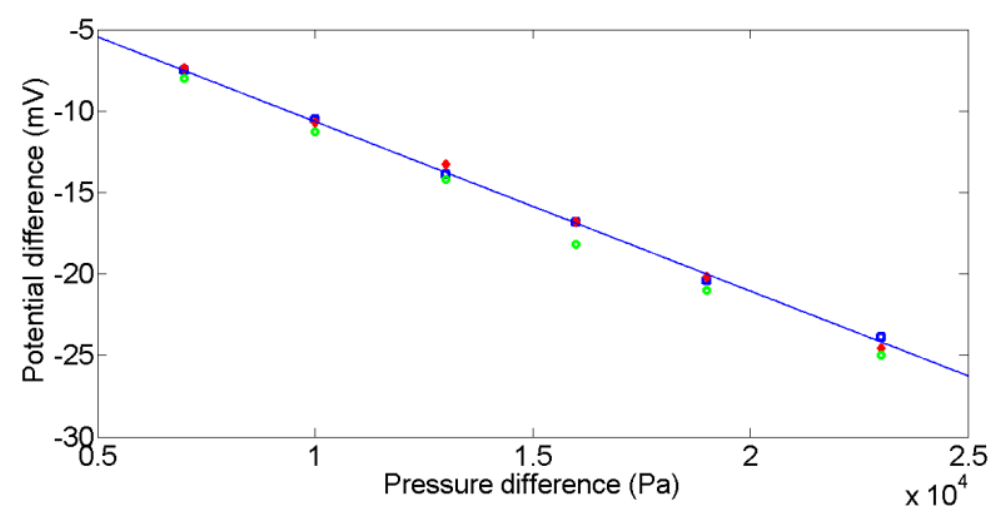

Figure 6. Streaming potential as a function of pressure difference for the sample $\mathrm{S} 3$ and electrolyte $\mathrm{KCl}$ at a concentration of $2.5 \times 10^{-3} \mathrm{M}$. Three representative measurements are shown. 


\subsection{Streaming potential measurement}

The experimental setup for the streaming potential measurement is shown in Fig. 5. The solution is circulated through the samples until the electrical conductivity and $\mathrm{pH}$ of the solution reach a stable value measured by a multimeter (Consort C861). The $\mathrm{pH}$ values of equilibrium solutions are in the range 6.0 to 7.5. Electrical potential differences across the samples are measured by a high input impedance multimeter (Keithley Model 2700) connected to a computer and controlled by a Labview program (National Instruments). Pressure differences across a sample are measured by a highprecision differential pressure transducer (Endress and Hauser Deltabar S PMD75).

Table 2. The streaming potential coefficient (in $\mathrm{mV} / \mathrm{bar}$ ) for different electrolyte concentrations.

\begin{tabular}{|c|c|c|c|c|c|c|c|}
\hline Sample ID & Electrolyte & $10^{-4} \mathrm{M}$ & $5.0 \times 10^{-4} \mathrm{M}$ & $10^{-3} \mathrm{M}$ & $2.5 \times 10^{-3} \mathrm{M}$ & $5.0 \times 10^{-3} \mathrm{M}$ & $10^{-2} \mathrm{M}$ \\
\hline \multirow{4}{*}{$\mathrm{S} 1$} & $\mathrm{NaCl}$ & -1250 & -202 & -120 & -41 & -20 & \\
\hline & $\mathrm{NaI}$ & -1275 & -206 & -125 & -44 & -22 & \\
\hline & $\mathrm{KCl}$ & -1033 & -183 & -91 & -30 & -11 & \\
\hline & $\mathrm{KI}$ & -1233 & -200 & -118 & -38 & -19 & \\
\hline \multirow{4}{*}{ S2 } & $\mathrm{NaCl}$ & -1950 & -380 & -179 & -70 & -32 & \\
\hline & $\mathrm{NaI}$ & -2067 & -403 & -185 & -87 & -35 & \\
\hline & $\mathrm{KCl}$ & -1230 & -263 & -127 & -58 & -26 & \\
\hline & $\mathrm{KI}$ & -1700 & -350 & -170 & -60 & -27 & \\
\hline \multirow{4}{*}{ S3 } & $\mathrm{NaCl}$ & -2100 & -625 & -357 & -145 & -63 & -31 \\
\hline & $\mathrm{NaI}$ & -2466 & -763 & -400 & -160 & -77 & -34 \\
\hline & $\mathrm{KCl}$ & -1567 & -570 & -313 & -120 & -65 & -25 \\
\hline & KI & -1665 & -573 & -330 & -123 & -58 & -26 \\
\hline \multirow{4}{*}{ S4 } & $\mathrm{NaCl}$ & -4021 & -842 & -429 & -149 & -69 & -33 \\
\hline & $\mathrm{NaI}$ & -4067 & -850 & -435 & -151 & -81 & -36 \\
\hline & $\mathrm{KCl}$ & -2333 & -576 & -290 & -106 & -71 & -29 \\
\hline & KI & -3933 & -836 & -430 & -147 & -75 & -31 \\
\hline
\end{tabular}

The way used to collect the SPC is already described in [23] and reference therein. Streaming potential across the sample $(\Delta \mathrm{V})$ is measured as a function of applied pressure difference $(\Delta \mathrm{P})$. The SPC is then obtained as the slope of the straight line (see Fig. 6). Three measurements are performed to find the average value of the SPC. The SPC for all samples at different electrolyte concentrations is shown in Table 2 except for two samples $\mathrm{S} 1$ and $\mathrm{S} 2$ at electrolyte concentration of $10^{-2} \mathrm{M}$. Because these samples are very permeable, they need a very large flow rate to generate measurable electric potentials at high electrolyte concentration. The maximum error of the SPC is $10 \%$. It is found that the SPC is negative for all samples and all electrolytes used in this work.

\section{Results and discussion}

From Table 2, the dependence of the SPC on types of electrolyte is shown in Fig. 7 for a representative sample (for example, sample S3). Fig. 7 shows that the magnitude of the SPC decreases with increasing electrolyte concentration for all samples and all electrolytes as reported in literature [e.g., 7, 8]. The experimental result also shows that the SPC mostly depend on types of cation in electrolytes. The dependence of the SPC on types of cation can be qualitatively explained by the difference in the binding constant of cations. For example, the binding constant of $\mathrm{K}^{+}$is larger than $\mathrm{Na}^{+}$[7]. Therefore, at the same ionic strength more cations of $\mathrm{K}^{+}$are absorbed on the negative solid surface than cations of $\mathrm{Na}^{+}$. This makes the electric potential on the shear plane (the zeta potential) 
smaller in the electrolyte containing cations of $\mathrm{K}^{+}$than that in the electrolyte containing cations of $\mathrm{Na}^{+}$. Therefore, cations have effect on the zeta potential and on the SPC. Namely, the SPC in magnitude is larger in the electrolyte containing cations of $\mathrm{Na}^{+}$than that in the electrolyte containing cations of $\mathrm{K}^{+}$. The experimental results also show that anions have only a small effect on the SPC as observed in [24].

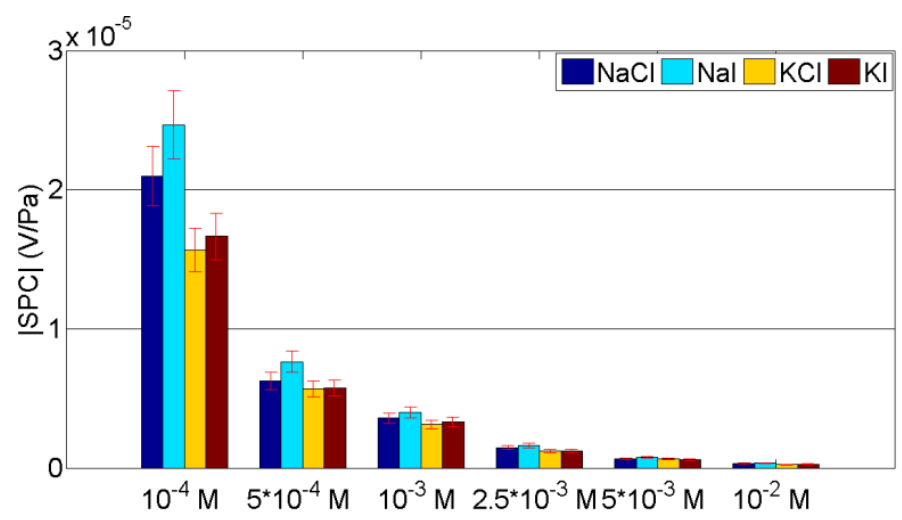

Figure 7. The magnitude of the SPC as a function of electrolyte concentration for different electrolytes and for the sample S3.

Fig. 8 shows the variation of the SPC with samples for electrolyte NaI, for example. It is seen that at a given electrolyte concentration, the SPC depends on the samples. Namely, the magnitude of the SPC increases in the order from S1, S2, S3 and S4 respectively. This may be due to the variation of effective conductivity with particle size of the samples and the difference in the zeta potential between the samples.

From Table 2, the magnitude of the SPC as a function of electrolyte concentration is plotted for all samples saturated by different electrolytes (see Fig. 9). By fitting the experimental data shown by the solid line in Fig. 9, the empirical relation between the SPC in magnitude and electrolyte concentration is obtained as

$$
C_{S}=\frac{2.0 \times 10^{-9}}{C_{f}}(\mathrm{~V} / \mathrm{Pa})
$$

where $C_{f}$ is electrolyte concentration.

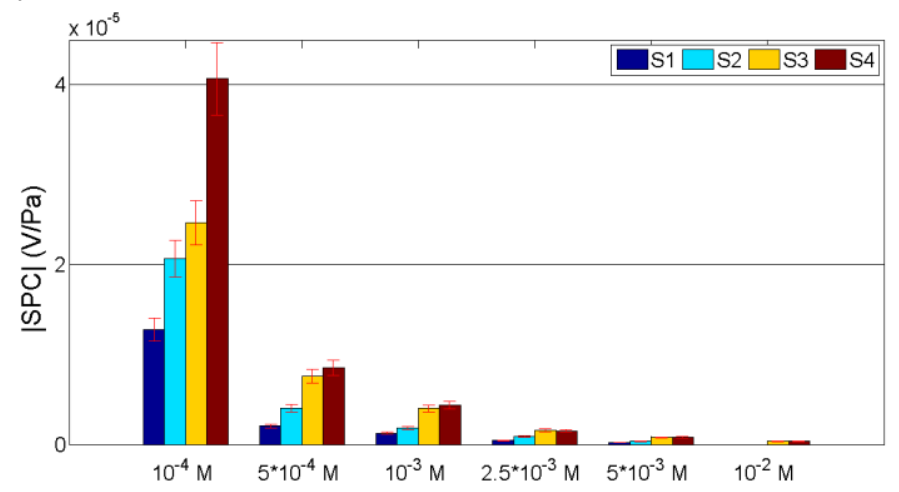

Figure 8. The magnitude of the SPC as a function of electrolyte concentration for different samples saturated by electrolyte $\mathrm{NaI}$. 
Expression in Eq. (12) has the similar form as the empirical expression $C_{S}=1.36 \times 10^{-9} / C_{f}^{0.9123}$ obtained by Jaafar et al. [9] by fitting experimental data collected for sandstone, sand, silica nanochannels, Stainton, and Fontainebleau with electrolytes of $\mathrm{NaCl}$ and $\mathrm{KCl}$ at $\mathrm{pH}=6-8$. Additionally, by fitting experimental data on sand saturated by $\mathrm{NaCl}$ at $\mathrm{pH}=7-8$ which are available in literature, Jouniaux and Ishido [8] obtain the expression $C_{S}=1.2 \times 10^{-8} / \sigma_{f}$ ( $\sigma_{f}$ is the fluid conductivity). The relation between fluid conductivity of a $\mathrm{NaCl}$ solution and electrolyte concentration in the range $10^{-6} \mathrm{M}<C_{f}<1 \mathrm{M}\left(15^{\circ} \mathrm{C}<\right.$ temperature $\left.<25^{\circ} \mathrm{C}\right)$ is given as $\sigma_{f}=10 C_{f}$ [25]. Therefore, we get the expression $C_{S}=1.2 \times 10^{-9} / C_{f}$ based on [8] and that is also similar to Eq. (12). The prediction of SPC as a function of electrolyte concentration from the empirical expressions of $[8,9]$ is also shown in Fig. 9 (see the dashed lines). It is seen that the predictions from [8,9] have the same behavior as that obtained in this work but give smaller values of the SPC at a given electrolyte concentration. The reason for the deviation between the empirical expressions may be due to dissimilarities of fluid conductivity, fluid $\mathrm{pH}$, mineral composition of porous media, temperature etc.

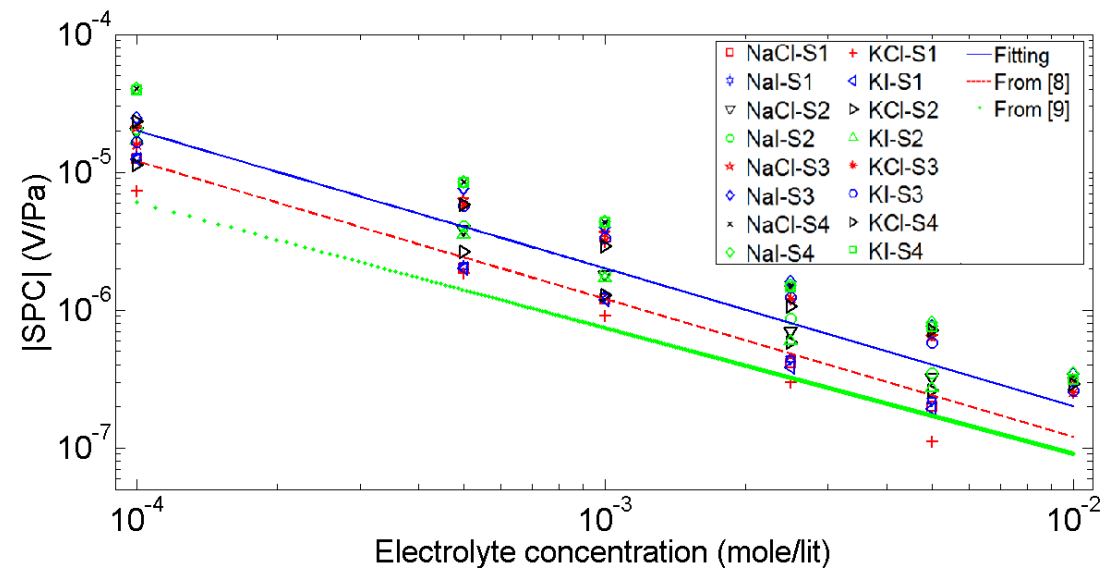

Figure 9. SPC in magnitude as a function of electrolyte concentration for different samples (S1, S2, S3 and S4) and different electrolytes ( $\mathrm{NaCl}, \mathrm{NaI}, \mathrm{KCl}$ and $\mathrm{KI}$ ). Symbols are experimental data. Solid line is the fitting line and two other dashed lines are predicted from [8] and [9].

\section{Conclusions}

The measurements of the streaming potential of four unconsolidated samples saturated with four monovalent electrolytes at different electrolyte concentrations have been performed. From the measured SPC, the empirical expression between the SPC and electrolyte concentration is obtained. The empirical expression is in good agreement with those reported in literature in which the SPC in magnitude is inversely proportional to electrolyte concentration. This work has added an additional empirical expression to the existing ones that allow us to predict the SPC from electrolyte concentration for silica-based samples saturated by monovalent electrolytes. Additionally, it is seen that the streaming potential coefficient depends on types of cation in electrolytes and on samples. The dependence of the streaming potential coefficient on types of cation is qualitatively explained by the difference in the binding constant for cation adsorption on the silica surfaces. The dependence of the streaming potential coefficient on samples is due to the variation of effective conductivity and the zeta potential between samples. 


\section{Acknowledgments}

The first author would like to thank Vietnam National Foundation for Science and Technology Development (NAFOSTED) under grant number 103.99-2016.29 for the financial support.

\section{References}

[1] B. Wurmstich, F. D. Morgan, Geophysics 59 (1994) 46-56.

[2] R. F. Corwin, D. B. Hoovert, Geophysics 44 (1979) 226-245.

[3] F. D. Morgan, E. R. Williams, T. R. Madden, Journal of Geophysical Research 94 (1989) 12.449-12.461.

[4] H. Mizutani, T. Ishido, T. Yokokura, S. Ohnishi, Geophys. Res. Lett. 3 (1976).

[5] M. Trique, P. Richon, F. Perrier, J. P. Avouac, J. C. Sabroux, Nature (1999) 137-141.

[6] A. A. Ogilvy, M. A. Ayed, V. A. Bogoslovsky, Geophysical Prospecting 17 (1969) 36-62.

[7] P. W. J. Glover, E. Walker, and M. D. Jackson, Geophysics 77 (2012) D17-D43.

[8] L. Jouniaux and T. Ishido, International Journal of Geophysics, vol. 2012, Article ID 286107, 16 pages, 2012. doi:10.1155/2012/286107.

[9] Vinogradov, J., M. Z. Jaafar, and M. D. Jackson, Journal of Geophysical Research Atmospheres 115 (2010) B12204.

[10] O. Stern, Z. Elektrochem 30 (1924) 508-516.

[11] T. Ishido, H. Mizutani, Journal of Geophysical Research 86 (1981) 1763- 1775.

[12] H. M. Jacob, B. Subirm, Electrokinetic and Colloid Transport Phenomena, Wiley-Interscience, 2006.

[13] K. E. Butler, Seismoelectric effects of electrokinetic origin, PhD thesis, University of British Columbia, 1996.

[14] H. Hase, T. Ishido, S. Takakura, T. Hashimoto, K. Sato, Y. Tanaka, Geophysical Research Letters 30 (2003) 3197-3200.

[15] R. J. Hunter, Zeta Potential in Colloid Science, Academic, New York, 1981.

[16] L. Onsager, Physical Review 37 (1931) 405-426.

[17] S. Dukhin, V. Shilov, Dielectric Phenomena and the Double Layer in Disperse Systems and Polyelectrolytes, John Wiley and Sons, New York, 1974.

[18] L. Jouniaux, M. L. Bernard, M. Zamora, J. P. Pozzi, Journal of Geophysical Research B 105 (2000) 8391-8401.

[19] Luong Duy Thanh, Rudolf Sprik, VNU Journal of Science: Mathematics-Physics 32 (2016) 22-33.

[20] Paul W. Glover and Nicholas Déry. Geophysics 75(2010), F225-F241.

[21] S. F. Alkafeef and A. F. Alajmi, Colloids and Surfaces A 289 (2006) 141-148.

[22] P. N. Sen, C. Scala, and M. H. Cohen, Geophysics 46 (1981) 781-795.

[23] Lưong Duy Thanh, Rudolf Sprik, VNU Journal of Science: Mathematics and Physics 31 (2015) 56-65.

[24] Luong Duy Thanh and Rudolf Sprik (2016). "Zeta potential in porous rocks in contact with monovalent and divalent electrolyte aqueous solutions" geophysics, 81(4), D303-D314.

[25] P. N. Sen and P. A. Goode, Geophysics 57 (1992) 89-96. 